\title{
Geometrical Resonance Conditions for THz Radiation from the Intrinsic Josephson Junctions in $\mathrm{Bi}_{2} \mathrm{Sr}_{2} \mathrm{CaCu}_{2} \mathrm{O}_{8+\delta}$
}

\author{
Manabu Tsujimoto, Kazuhiro Yamaki, Kota Deguchi, Takashi Yamamoto, \\ Takanari Kashiwagi, Hidetoshi Minami, Masashi Tachiki, and Kazuo Kadowaki \\ Institute of Materials Science, Graduate School of Pure 83 Applied Sciences, \\ University of Tsukuba, 1-1-1, Tennodai, Tsukuba, Ibaraki 305-8573, Japan \\ Richard A. Klemm \\ Department of Physics, University of Central Florida, Orlando, Florida 32816, USA
}

(Dated: October 29, 2018)

\begin{abstract}
Subterahertz radiation emitted from a variety of short rectangular-, square-, and disk-shaped mesas of intrinsic Josephson junctions fabricated from a $\mathrm{Bi}_{2} \mathrm{Sr}_{2} \mathrm{CaCu}_{2} \mathrm{O}_{8+\delta}$ single crystal was studied from the observed $I-V$ characteristics, far-infrared spectra, and spatial radiation patterns. In all cases, the radiation frequency satisfies the conditions both for the ac Josephson effect and for a mesa cavity resonance mode. The integer higher harmonics observed in all spectra imply that the ac Josephson effect plays the dominant role in the radiation.
\end{abstract}

PACS numbers: 07.57.Hm, 74.50.+r, 85.25.Cp

After the first report of intense continuous terahertz $\left(\mathrm{THz}, 1 \mathrm{THz}=10^{12} \mathrm{~Hz}\right)$ electromagnetic $(\mathrm{EM})$ waves emitted from the intrinsic Josephson junctions (IJJs) in the high temperature superconductor (HTSC) $\mathrm{Bi}_{2} \mathrm{Sr}_{2} \mathrm{CaCu}_{2} \mathrm{O}_{8+\delta}(\mathrm{Bi}-2212)$ by Ozyuzer et al. [1] with remarkably higher intensity than previously generated from Josephson junctions [2], a great deal of interest has been drawn not only to the physical mechanism of the radiation but also to the possible variety of applications in the vast fields of science and technology. Nondestructive inspections, medical diagnostics, high speed communications, imaging technologies for security and defense, etc., are potential candidates among them. Presently available $\mathrm{THz}$ sources such as those using parametric generation or pulsed-current methods based on semiconductor and/or laser technology are rather weak in output power, and are mostly pulsed with incoherent waves. Although it is possible to generate output power of even $\sim \mu \mathrm{W}$ in a two-dimensional array of Josephson junctions [3], frequencies above a few hundred $\mathrm{GHz}$ cannot be achieved with conventional superconductors due to the small energy gap of a few meV $(1 \mathrm{meV} \Leftrightarrow 483.5979 \mathrm{GHz})$. However, single crystalline Bi-2212 has an approximately 10 times larger energy gap, so that in principle it can be employed to reach frequencies of several THz. Furthermore, it was shown [1] that an entirely new mechanism for $\mathrm{THz}$ EM wave generation occurs in Bi-2212 due to its highly anisotropic layered structure of IJJs [4].

Here, we present direct unambiguous evidence that the ac Josephson effect is the driving mechanism for the radiation, in which the fundamental frequency $f_{1}$ equals the frequency $f_{J}=2 \mathrm{eV} /(N h)$ of the $a c$ Josephson effect, where $V$ is the applied $d c$ voltage across the $N$ IJJs, $e$ is the electric charge, and $h$ is Planck's constant. $f_{1}$ is also in resonance with a frequency $f_{m p}$ of a thin EM cavity mode, which is inversely proportional to the mini-

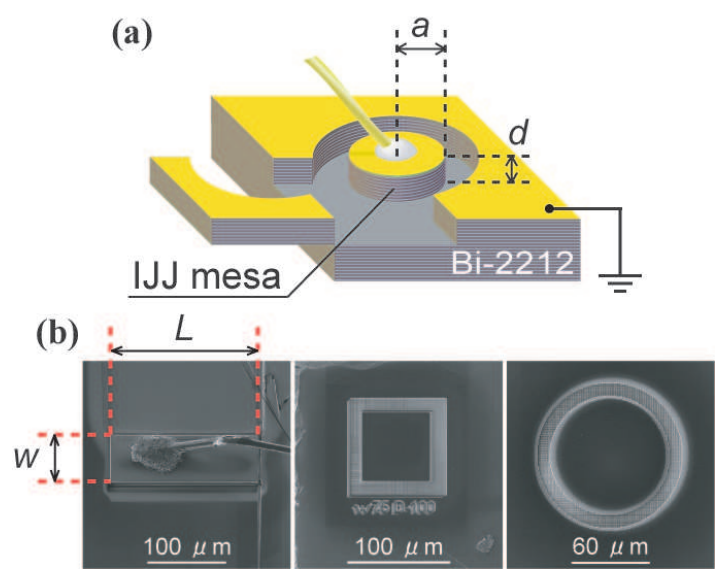

FIG. 1: (a) Sketch of a cylindrical disk IJJ mesa. The grazing angle forming the groove of $10 \mu \mathrm{m}$ width is approximately $5^{\circ}$. (b) Scanning ion microscope images of a rectangular (left), square (center), and disk (right) mesa.

mal mesa cross-section dimension. We demonstrate $\mathrm{THz}$ (or sub-THz) radiation from mesas of various geometrical shapes, using rectangular-, square-, and cylindrical disk-shaped mesas fabricated by focused ion beam (FIB) milling. We first examine the observed radiation frequencies as functions of the geometrical sizes of the mesas, in order to analyze the data by the above two radiation conditions [1, 5]. The cylindrical geometry is particularly interesting for understanding the radiation, because the standing EM cavity mode frequencies $f_{m p}^{c}$ are determined by the zeroes of the first derivatives of standard Bessel functions, which are incommensurate with the harmonic ac Josephson frequency spectrum $n f_{J}$ for integer $n[6]$. Hence, only one frequency can equal both a cylindrical cavity mode frequency and an $a c$ Josephson frequency. In contrast, for rectangular mesas, each member of a subset 
TABLE I: List of the parameters of the IJJ mesa samples. See text.

\begin{tabular}{|c|c|c|c|c|c|c|c|}
\hline No. & Geometry & $a[\mu \mathrm{m}]$ & $w(L)[\mu \mathrm{m}]$ & $d[\mu \mathrm{m}]$ & $T_{c}\left(\Delta T_{c}\right)[\mathrm{K}]$ & $\delta T[\mathrm{~K}]$ & $j_{c}\left[\mathrm{~A} / \mathrm{cm}^{2}\right]$ \\
\hline$\overline{\mathrm{D} 1}$ & Disk & $33.9-38.9$ & & 1.4 & $88.3(1.9)$ & $20-50$ & 200 \\
\hline D2 & Disk & $48.9-51.5$ & & 1.5 & $88.5(2.0)$ & $35-45$ & 55 \\
\hline D3 & Disk & $61.5-65.0$ & & 1.6 & $85.9(4.0)$ & $10-50$ & 190 \\
\hline S1 & Square & & $66.7-75.7,70.9-81.6$ & 1.7 & $87.7(1.1)$ & $30-40$ & 180 \\
\hline $\mathrm{R} 1$ & Rectangle & & $59.7-64.4(200)$ & 1.5 & $82.3(2.5)$ & $10-25$ & 170 \\
\hline
\end{tabular}

(a)

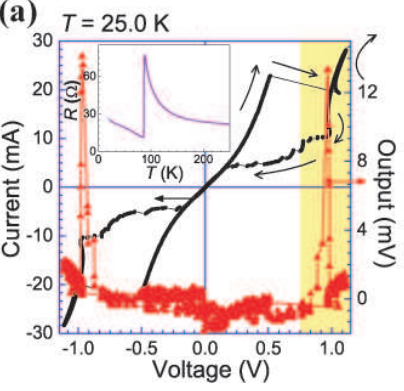

(b)

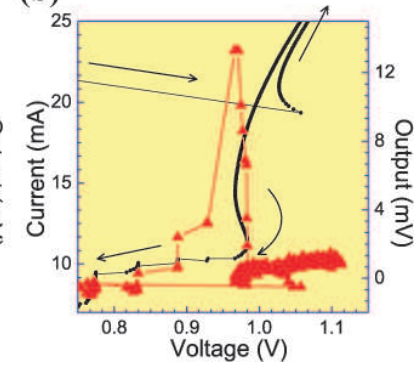

FIG. 2: (a) The $I-V$ characteristics (left scale) and output radiation intensity detected by the Si-bolometer (right scale) from disk mesa D3 at the bath temperature $T=25.0 \mathrm{~K}$ of its maximal radiation intensity are shown. The inset shows the $c$-axis $R$ - $T$ curve. (b) Details of the shaded high bias region in Fig. 2(a) where the emission is observed.

of higher cavity modes of frequencies $f_{m p}^{\mathrm{r}}$ can be degenerate with an harmonic of the fundamental $a c$ Josephson frequency $f_{J}[6]$. For the rectangular mesas used in all previous studies [1, 5, 7], it was therefore not possible to unambiguously determine the primary mechanism of the radiation. Comparisons of the angular distribution of the observed radiation with that predicted can also provide information as to the relative importance of the two mechanisms for the radiation $[6,8,8]$.

The single crystals of $\mathrm{Bi}-2212$ used in the present studies were prepared by a floating zone technique [10]. The as-grown single crystals were annealed at $650{ }^{\circ} \mathrm{C}$ for $24 \mathrm{~h}$ in argon gas mixed with $0.1 \%$ oxygen in order to obtain underdoped crystals. The temperature $T$ dependence of the $c$-axis resistance ( $R$ - $T$ curve) shows the behavior typical of slightly underdoped Bi-2212 crystals with a critical temperature $T_{c} \approx 87 \mathrm{~K}$. A small piece of a cleaved crystal $\sim 50 \mu \mathrm{m}$ thick was glued onto a sapphire substrate by silver paste. Next, silver and gold thin layers were evaporated onto the surface. Then, a groove of width $10 \mu \mathrm{m}$ and depth of $\sim 2 \mu \mathrm{m}$ was patterned by FIB milling, making an islandlike terrace as sketched in Fig. 1(a). At the end of the process, a $10 \mu \mathrm{m}$ gold wire electrode was connected to the center of the top metal layer of the mesa by silver paste. The sample dimensions and profile curves were measured by an atomic force microscope and the results are presented in Table 【. The cross-sectional profiles are considerably slanted and rounded at the edges, resulting in approximately trapezoidal shapes (not shown

here) [5, 9]. Typically the top cross-section is approximately 10-20\% smaller than the bottom one. Thin mesas with three different cross-sectional shapes were studied: one rectangular, one square, and three circular disks with properties listed in Table [ The total number $N \sim 1000$ of IJJs is roughly estimated from each mesa height $d$.

The $I-V$ characteristics of disk mesa D3, measured by sweeping the bias $d c$ current $I$ along the $c$-axis at the bath temperature $T=25.0 \mathrm{~K}$, are displayed in Fig. 2(a) together with the radiation intensity detected by the Sibolometer. In Fig. 2(b) the radiation region is shown in detail in an expanded scale. All mesas that emit THz radiation studied to date have a critical current density $j_{\mathrm{c}}$ ranging between 55 and $200 \mathrm{~A} / \mathrm{cm}^{2}$ as listed in Table [. The strongest radiation occurs on the return branch of the outermost $I$ - $V$ curve. In most cases, the $\mathrm{THz}$ radiation was observed in a very narrow voltage range. For example, emission occurs for sample D3 between 0.96 and $0.98 \mathrm{~V}$, corresponding to $I \approx 10.8 \mathrm{~mA}$. Then, the radiation suddenly stops due to a jump to another $I$ $V$ characteristic branch. The radiation power density at the detector just before jumping is estimated to be $1.3 \mathrm{nW} / \mathrm{cm}^{2}$, the same order of magnitude as obtained previously from rectangular mesas [5]. If the interbranch jump did not occur, the radiation intensity would likely have grown much stronger [9].

It is also interesting to note that the emission usually occurs within a sample-dependent range $\delta T$ of the base temperature of the mesa, as summarized in Table I. For example, mesa D3 emits between 10 and $50 \mathrm{~K}$. Since the constant $I \approx 11 \mathrm{~mA}$ is fed into mesa D3, it is inevitably heated at a rate of about $11 \mathrm{~mW}$, corresponding to the enormous heating power density of $8.3 \mathrm{~kW} / \mathrm{cm}^{3}$. This huge heating power density cannot be removed quickly enough from the mesa to maintain equilibrium, resulting in a considerable rise of the mesa temperature. This local heating may induce a chaotic nonequilibrium state and may adversely affect the $\mathrm{THz}$ radiation [11, 12]. Since the heat conduction is progressively worse as $T$ is lowered 13 , 14] and the gap vanishes as $T \rightarrow T_{c}$, these features may account for the peculiar temperature dependence of the radiation intensity.

In Fig. 3(a), the far-infrared spectra of the $\mathrm{THz}$ radiation from the disk mesas, as measured by a Fourier transform infrared (FTIR) spectrometer, are shown. In the inset to Fig. 3(a), the fundamental radiation frequency 
$f_{1}=f_{J}$ is plotted as a function of $1 /(2 a)$, where $a$ is the mesa radius. The dashed line represents the resonance frequency $f_{J}=f_{11}^{\mathrm{c}}=\chi_{11} c_{0} /(2 \pi \sqrt{\epsilon} a)$, where $\chi_{11}=1.841$ for the TM $(1,1)$ mode, $\epsilon$ is the dielectric constant of Bi2212 , and $c_{0}$ is the speed of light in vacuum, predicted by the cavity resonance model [15], assuming the cylindrical cavity boundary condition $\left.H_{\phi}\right|_{\rho=a}=\left.\left(\partial E_{z} / \partial \rho\right)\right|_{\rho=a}=0$. The radiation frequency $f_{1}$ is clearly proportional to $1 /(2 a)$. The data were fitted with $\epsilon=17.6$, in good agreement with previous results [5, 9]. Note that this $\epsilon$ value is about $50 \%$ larger than that obtained from infrared spectroscopy [16]. In addition, for each disk mesa, the second harmonic at $f_{2}=2 f_{J}$ is clearly visible, and these frequencies are easily distinguishable from those of the nearest higher disk cavity modes [6], providing unambiguous experimental evidence that the uniform $a c$ Josephson current is the primary radiation source.

In order to further understand the excitation mode inside the disk mesa, the radiation intensity $\mathcal{I}$ was measured at various detection angles $\theta$, relative to the normal to the mesa. In Fig. 3(b), $\mathcal{I}(\theta)$ for disk mesa D3 is presented. The shadowing effect of the radiation from the superconducting Bi-2212 crystal wall outside the groove is expected to be negligibly small to first approximation. Therefore, the following characteristic features are noted: First, $\mathcal{I}(\theta)$ is strongly anisotropic, having a maximum around $\theta=\theta_{\max }=20-35^{\circ}$ from the top $\left(\theta=0^{\circ}\right)$, where a local minimum occurs with intensity ratio $\mathcal{I}\left(\theta=0^{\circ}\right) / \mathcal{I}_{\max }\left(\theta=20^{\circ}\right)=0.50-0.65$. This shallow minimum feature is almost the same as for rectangular mesas, although $\theta_{\max }$ is somewhat less than the corresponding rectangular mesa value [9]. Second, $\mathcal{I}(\theta)$ rapidly diminishes as $\theta$ approaches $90^{\circ}$. $\mathcal{I}_{\text {cav }}(\theta)$ calculated [6] by assuming radiation from the $\operatorname{TM}(1,1)$ cavity resonance mode alone is shown by the dashed black curve in Fig. 3 (b). Clearly, the calculated $\mathcal{I}_{\text {cav }}(\theta)$ does not fit the experimental data, especially near to $\theta=0^{\circ}$, where $\mathcal{I}_{\text {cav }}(\theta)$ is a maximum [6]. This disagreement can be reduced by introducing a superposition of the radiation from the uniform $a c$ Josephson current source with the same Josephson frequency, as described for rectangular mesas [6, 9]. The experimental data are better fitted by the dual-source model $\mathcal{I}_{\text {dual }}(\theta)$ with mixing parameter $\alpha=1.44[6]$, corresponding to $58 \%$ of the radiation arising from the uniform $a c$ Josephson current source, as shown by the solid orange curve in Fig. 3(b).

It is significant that the intensity from the uniform source is comparable to that of the fundamental cavity mode source, as in rectangular mesas [9]. Since the fundamental cavity mode radiation is enhanced by the cavity quality Q-value, a similar enhancement must occur for the uniform source radiation [17]. This suggests that the radiation from the uniform ac Josephson current in the $N$ junctions is coherent, amplifying the output by a factor of order $N^{2}[3]$. This interpretation is strongly supported by the observation of only integral higher harmonics of (a)
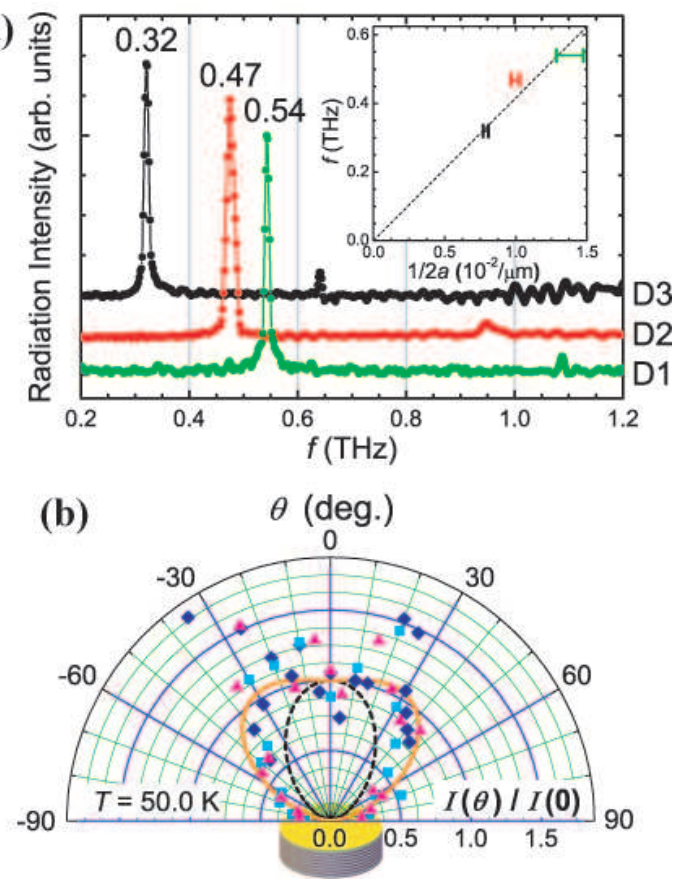

FIG. 3: (a) The radiation spectra measured by the FTIR spectrometer for three disk mesas. The inset shows the observed frequencies versus $1 /(2 a)$. The error bars reflect the trapezoidal cross-section profile. The dashed line represents the resonance frequency expected from the cylindrical cavity TM $(1,1)$ mode with $\epsilon=17.6$. (b) Polar plot of the radiation intensity for disk mesa D3. Different symbols correspond to different runs. The solid orange and dashed black curves are the best fit to the dual-source model and its cavity component, respectively.

the fundamental frequencies shown in Fig. 3(a). Neither higher cylindrical cavity excitation frequencies of the Bessel type nor subharmonics were observed. The higher harmonics are naturally present in the uniform Josephson current, which radiates coherently, but the nonuniform part of which only can excite one cylindrical cavity mode. This experimental evidence clarifies unambiguously that the $\mathrm{THz}$ radiation is mainly generated by the uniform mode of the ac Josephson current, in sharp contrast to a number of theoretical predictions [8, 18 20]. Note that although the harmonics arise solely from the uniform $a c$ Josephson current source, the larger intensity fundamental frequency is a mixture of both the uniform ac Josephson and nonuniform cavity sources. Further studies of the polarization, coherence, and spatial radiation patterns of the higher harmonics could provide supporting information for these conclusions.

The resonance frequencies of the $\operatorname{TM}(m, p)$ modes of a rectangular cavity of width $w$ and length $L$ are $f_{J}=f_{m p}^{\mathrm{r}}=\left(c_{0} / 2 \sqrt{\epsilon}\right) \sqrt{(m / L)^{2}+(p / w)^{2}}$ [9], whereas for cylindrical cavities, they are $f_{J}=f_{m p}^{c}=$ $\chi_{m p} c_{0} /(2 \pi \sqrt{\epsilon} a)$, where $\chi_{m p}$ is the $p$ th zero of the derivative of $J_{m}(z)$, the standard Bessel function of order $m[\underline{6}$. 
Theoretically, the fundamental modes are the TM $(1,0)$ and TM $(1,1)$ modes for rectangular and cylindrical cavities, respectively. Although this is consistent with experiment for cylindrical mesas, it is very curious that the TM $(1,0)$ mode for rectangular mesas was never observed in experiment 1, 5, 9]. Since the mesa may be considerably heated by the $d c$ current, it is very likely that the inhomogeneous heat distribution inside it prevents standing EM wave formation, particularly along the longer rectangular dimension. However, such hot spots with $T$ even exceeding $T_{c}$ observed by Wang et al. [11] do not seem to be a problem in our experiments, since the fundamental frequencies of our disk and square mesas excellently obey the linear relation of the cavity resonance frequencies with $1 / a$ and $1 / w$, respectively. The discrepancies in relatively long rectangular mesas with $w \ll L=300$ $400 \mu \mathrm{m}$ may have a different origin. In order to check this, we fabricated rectangular mesas with smaller $L / w$ ratios, as shown in the left and center scanning ion microscope images in Fig. 1(b). The spectroscopic emission

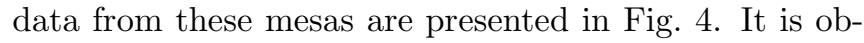

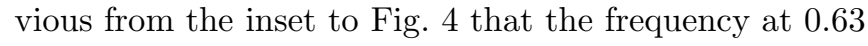
$\mathrm{THz}$ of the rectangular mesa $\mathrm{R} 1$ with $L / w \approx 3.1-3.3$ obeys the TM $(0,1)$ cavity resonance mode very well, but definitely not the TM $(1,0)$ resonance mode. These rather surprising observations strongly suggest that the formation of EM standing waves are restricted by other as yet undetermined reasons, and may not be excited below some cutoff frequency. We propose that this cutoff frequency may be the Josephson plasma frequency $f_{p}=c_{0} /\left(2 \pi \sqrt{\epsilon} \lambda_{c}\right)$, where $\lambda_{c}$ is the $c$-axis superconducting penetration depth.

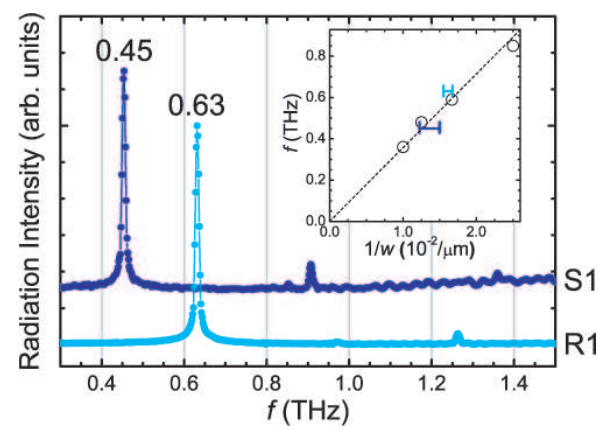

FIG. 4: The radiation spectra for the square (S1) and rectangular (R1) mesas. The inset shows the observed frequencies versus the narrower width inverse $1 / w$, where the circles are previous data [9]. The black dashed line represents the resonance frequency expected for the rectangular cavity $\operatorname{TM}(0,1)$ mode with $\epsilon=17.6$.

In summary, we studied the $\mathrm{THz}$ radiation generated from IJJ mesas of Bi-2212 with different geometrical shapes. Our experimental results clearly demonstrate the validity of the cavity resonance model for the fundamental frequency mode of thin square and cylindrical mesas. The spatial radiation pattern cannot be ex- plained by the cavity resonance model alone, but requires a substantial contribution from the uniform ac Josephson current source. More importantly, the frequency spectra obtained exhibit higher integral harmonics of the fundamental $f_{1}$, which cannot be obtained from higher cavity resonance modes in cylindrical cavities, providing unambiguous experimental evidence that the uniform $a c$ Josephson current is the primary radiation source. Although heating effects may significantly alter the $I-V$ characteristics, they do not greatly affect the two necessary radiation conditions: the $a c$ Josephson relation, $f_{1}=f_{J}=2 \mathrm{eV} / \mathrm{Nh}$, and the geometrical cavity resonance condition $f_{1}=f_{11}^{\mathrm{c}}$ or $f_{1}=f_{01}^{\mathrm{r}}$, for cylindrical or rectangular cavities, respectively, each inversely proportional to the minimal cross-sectional dimension. We propose a further radiation condition that $f_{1}>f_{p}$, the Josephson plasma frequency.

The authors deeply thank X. Hu, S. Lin, A. Koshelev, M. Matsumoto, T. Koyama, M. Machida, S. Fukuya, and K. Ivanovic for valuable discussions and Y. Ootuka, A. Kanda, I. Kakeya, H. Yamaguchi, N. Orita, T. Koike, and B. Markovic for their technical assistance. This work has been supported in part by CREST-JST (Japan Science and Technology Agency), WPI (World Premier International Research Center Initiative)-MANA (Materials Nanoarchitectonics) project (NIMS) and Strategic Initiative category (A) at the University of Tsukuba.

[1] L. Ozyuzer et al., Science 318, 1291 (2007).

[2] D. N. Langenberg et al., Phys. Rev. Lett. 15, 294 (1965).

[3] P. Barbara et al., Phys. Rev. Lett. 82, 1963 (1999).

[4] R. Kleiner et al., Phys. Rev. Lett. 68, 2394 (1992).

[5] K. Kadowaki et al., Physica C 468, 634 (2008).

[6] R. A. Klemm and K. Kadowaki, (unpublished); arXiv:0908.4104

[7] H. Minami et al., Appl. Phys. Lett. 95, 232511 (2009).

[8] X. Hu and S. Lin, Phys. Rev. B 78, 134510 (2008).

[9] K. Kadowaki et al., J. Phys. Soc. Jpn. 79, 023703 (2010).

[10] T. Mochiku and K. Kadowaki, Physica C 235-240, 523 (1994).

[11] H. B. Wang et al., Phys. Rev. Lett. 102, 017006 (2009).

[12] C. Kurter et al., IEEE Trans. Appl. Supercond. 19, 428 (2009).

[13] T. Yasuda, M. Tonouchi, and S. Takano, Physica C 289, 109 (1997).

[14] J. C. Fenton et al., Appl. Phys. Lett. 80, 2535 (2002).

[15] A. G. Deneryd, IEEE Trans. Antennas Propagat. AP27, 660 (1979).

[16] S. Tajima et al., Phys. Rev. B 48, 16164 (1993).

[17] M. Tachiki, S. Fukuya, and T. Koyama, Phys. Rev. Lett. 102, 127002 (2009).

[18] A. E. Koshelev and L. N. Bulaevskii, Phys. Rev. B 77, 014530 (2008).

[19] S. Lin, X. Hu, and M. Tachiki, Phys. Rev. B 77, 014507 (2008)

[20] S. Lin and X. Hu, Phys. Rev. Lett. 100, 247006 (2008). 